G. Ikegami

Nagoya Math. J.

Vol. 55 (1974), 161-179

\title{
ON WEAK CONCEPTS OF STABILITY
}

\author{
GIKŌ IKEGAMI
}

\section{§ 1. Introduction.}

The manifold in this paper is assumed to be connected differentiable of class $C^{\infty}$. Let $\mathscr{D}^{r}(M)$ and $\mathfrak{X}^{r}(M)$ be the set of all diffeomorphisms and vector fields of class $C^{r}$ on a manifold $M$ with Whitney $C^{r}$ topology, respectively. In [2], the concept of weak stability is defined. The definition is equivalent to the following ((2.1) of this paper); $f \in \mathscr{D}^{r}(M)$ or $X \in \mathfrak{X}^{r}(M)$ is weakly (allowably) stable if and only if there is a neighborhood $U$ of $f$ or $X$ in $\mathscr{D}^{r}(M)$ or $\mathfrak{X}^{r}(M)$ such that for any (a suitable) $g$ or $Y \in U$ the set of all elements topologically equivalent to $g$ or $Y$ is dense in $U$, respectively. Here, $f, g \in \mathscr{D}^{r}(M)$ are said to be topologically equivalent if they are topologically conjugate and $X, Y \in \mathfrak{X}^{r}(M)$ are said to be topologically equivalent if there is a homeomorphism mapping any trajectory of $X$ onto a trajectory of $Y$ preserving the orientations of the trajectories. Similarly, weak $\Omega$-stability is defined for $f$ and $X$.

All weakly ( $(\Omega-)$ stable systems compose an open set. The set of all systems which are weakly $(\Omega-)$ stable but not structurally (or $\Omega-$ ) stable is also an open set. In [2] it is shown that in case of the non-wandering set being finite the weak stability of a diffeomorphism of a compact manifold implies the structural stability. The nondensity of weakly stable diffeomorphisms and weakly $\Omega$-stable diffeomorphisms are shown in [2].

One aim of this paper is to prove some results ((2.2), (3.2), and (4.2)) about allowable stability which are similar to some results mentioned in [2] with or without proof. Another aim is to prove the existence of a vector field on the 2-plane $\boldsymbol{R}^{2}$ which is weakly stable but not structurally stable. This example is mentioned in [2] without proof.

\section{§ 2. Weak stability and allowable stability.}

Let $T$ be any topological space and $\sim$ be an equivalence relation 
between the elements of $T$. An element $x$ of $T$ is said to be stable with respect to the equivalence $\sim$ if there is a neighborhood $U$ of $x$ such that $x \sim y$ for any $y$ in $U$.

In [2] a concept of weak stability is defined for dynamical systems. It is defined as follows for topological space $T$. For $x, y$ in $T$ we denote $x \widetilde{\rightarrow}$, if for any neighborhood $V$ of $y$ in $T$ there is a neighborhood $U$ of $x$ in $T$ such that for any $x^{\prime}$ in $U$ there is $y^{\prime}$ in $V$ with $x^{\prime} \sim y^{\prime}$. We denote $x \simeq y$ if and only if $x \simeq y$ and $y \simeq x$. Then, $x \in T$ is said to be weakly stable with respect to the equivalence $\sim$ if there is a neighborhood $U$ of $x$ such that $x \simeq y$ for any $y$ in $U$.

The next property (2.1) gives an equivalent definition of weak stability.

(2.1) $x \in T$ is weakly stable if and only if there is a neighborhood $U$ of $x$ such that for any $y \in U$ the set of all elements equivalent to $y$ is dense in $U$.

Proof. If $x$ is weakly stable, there is a neighborhood $U$ of $x$ such that for any $y \in U y \simeq x$. It is sufficient to show that for any $z$ in $U$ and any neighborhood $V$ of $z$ there is $z^{\prime} \in V$ equivalent to $y$. Since $\simeq$ is an equivalence relation, $y \simeq x$ and $z \simeq x$ imply $y \simeq z$. Hence, $y \simeq z$. This shows that there is $z^{\prime}$ in $V$ such that $z^{\prime} \sim y$. The converse of (2.1) is clear.

This property (2.1) reminds us of a following weaker concept of stability than weak stability. $R$. Thom also suggested to the author the following concept of stability by a different definition in a discussion after the conference on manifolds and topology held in Tokyo. $x \in T$ is said to be allowably stable with respect to the equivalence $\sim$ if there is a neighborhood $U$ of $x$ with the following property; there is $x_{0}$ in $U$ such that the set of all elements equivalent to $x_{0}$ is dense in $U$. We say such element $x_{0}$ a dispersed element about the allowably stable element $x$.

By the definitions the set of all elements which are stable, weakly stable, or allowably stable is an open set. Moreover, the set of all elements which are weakly stable but not stable is an open set.

Let $\mathscr{D}^{r}(M)$ and $\mathfrak{X}^{r}(M)$ be the set of all diffeomorphism and vector fields, respectively, on a manifold $M$ with Whitney $C^{r}$ topology. For diffeomorphisms $f$ and $g$ in $\mathscr{D}^{r}(M) f$ and $g$ are said to be topologically 
equivalent (or conjugate) and denoted by $f \sim g$ if there is a homeomorphism $h$ on $M$ satisfying $f h=h g$. For $X$ and $Y$ in $\mathfrak{X}^{r}(M), X$ and $Y$ are said to be topologically equivalent and denoted by $X \sim Y$ if there is a homeomorphism mapping any orbit of $X$ onto an orbit of $Y$ preserving orientations of the orbits. Let $\Omega=\Omega(f)$ or $\Omega=\Omega(X)$ be the nonwandering set of $f$ or $X$. We denote $f \sim_{\Omega} g$ or $X \sim_{s} Y$ if $f \mid \Omega(f)$, $g \mid \Omega(g)$ or $X|\Omega(X), Y| \Omega(Y)$ are topologically equivalent by the similar sense as above. $f \in \mathscr{D}^{r}(M)$ and $X \in \mathfrak{X}^{r}(M)$ are said to be structurally $C^{r}$ stable, weakly $C^{r}$ stable, or allowably $C^{r}$ stable if they are stable, weakly stable, or allowably stable, with respect to topological equivalence, respectively. For $f$ and $X C^{r} \Omega$-stability, weak $C^{r} \Omega$-stability, and allowable $C^{r} \Omega$-stability are defined similarly using the equivalence $\sim_{\Omega}$.

(2.2) Proposition. Let $f$ be a $C^{r}$ diffeomorphism of a compact manifold, $1 \leqq r \leqq \infty$. If $f$ is allowably $C^{r}$ stable, then all periodic point of a dispersed diffeomorphism about $f$ is hyperbolic.

The proof is very similar to that of (2.4) in [2].

Proof of (2.2). Let $f_{0}$ be a dispersed diffeomorphism near $f$. Since $f$ is allowably stable, there is a $C^{r}$ neighborhood $U$ of $f$ such that the set of all diffeomorphisms topologically conjugate to $f_{0}$ is dense in $U$. Suppose that there is a periodic point $p$ of $f_{0}$ which is not hyperbolic. Let $n$ be the period of $p$.

The number of the periodic points of $n$ must be finite. In fact, assume that there are infinitely many such periodic points. By KupkaSmale, there is $g$ in $U$ such that $g$ has only hyperbolic periodic points and there is a neighborhood $V$ of $g$ such that for any $h \in V$ all periodic points of $h$ of period $n$ are hyperbolic. Hence, the number of periodic points of $h$ of period $n$ is finite. Since $f_{0}$ is dispersed, there is $h \in V$ with $f_{0} \sim h$. But the number of periodic points of period $n$ of $f_{0}$ is infinite. This is a contradiction.

By a small perturbation of $f_{0}$ only in a neighborhood of nonhyperbolic periodic points of period $n$ of $f_{0}$ we have $f_{0}^{+} \in U$ which has only hyperbolic periodic points of period $n$ so that (i) in a neighborhood of a nonhyperbolic periodic point $p$ of $f$ of period $n$ there is only one periodic point $p^{+}$of $f_{0}^{+}$of period $n$, and (ii) $p$ and $p^{+}$has the same number of eigenvalues $\lambda$ with $|\lambda|<1$, so the eigenvalues of $p$ with $|\lambda|=1$ 
are altered to that of $p^{+}$so that $|\lambda|>1$. Similarly we have $f_{0}^{-} \in U$ by altering the eigenvalues of $p$ with $|\lambda|=1$ to the eigenvalues of $p^{-}$so that $|\lambda|<1$. Since $f_{0}$ is dispersed and all the periodic points of period $n$ of $f_{0}^{+}$are hyperbolic, the numbers of the periodic points of period $n$ of $f_{0}$ and $f_{0}^{+}$are the same. Similar fact holds for $f_{0}^{-}$. There is a neighborhood $V^{+}$of $f_{0}^{+}$such that for any $g^{+} \in V^{+} f_{0}^{+}$and $g^{+}$have the same number of hyperbolic periodic points of period $n$ and of the same index. There is similar neighborhood $V^{-}$of $f_{0}^{-}$. Then, for any $g^{+} \in V^{+}$and $g^{-} \in V^{-}, g^{+} \nsim g^{-}$. This contradicts to the fact that all diffeomorphisms equivalent to $f_{0}$ are dense in $U$. Therefore, $f_{0}$ has only hyperbolic periodic points. This proves (2.2).

(2.3) CoRollaRY ((2.4) of [2]). Let $f$ be a $C^{r}$ diffeomorphism of a compact manifold, $1 \leqq r \leqq \infty$. If $f$ is weakly $C^{r}$ stable, then all periodic points of $f$ are hyperbolic.

\section{§. Suspensions and associated diffeomorphisms.}

The aim of this section is to prove theorem (3.2).

Let $\phi_{t}(x)$ be a $C^{r}$ flow associated to a vector field $X$ on a compact manifold, i.e. if we put $\phi_{t}(x)=x(t),(d / d t) x(t)=X(x(t))$ and $x(0)=x$. A $C^{r}$ cross-section of $\phi_{t}(x)$ is a compact $C^{r}$ submanifold $w$ with codimension 1 such that

(i) $W$ intersects every orbit,

(ii) the intersection of $W$ with each orbit is transversal,

(iii) if $x \in W$, there is a $t>0$ with $\phi_{t}(x) \in W$, and

(iv) if $x \in W$, there is a $t<0$ with $\phi_{t}(x) \in W$.

If $\phi_{t}(x)$ has a cross-section $W$, a diffeomorphism $f: W \rightarrow W$, called the associated diffeomorphism of $\phi_{t}(x)$, is defined by $f(x)=\phi_{t_{0}}(x)$ where $t_{0}$ is the smallest positive $t$ satisfying $\phi_{t}(x) \in W$. Conversely, suppose that a diffeomorphism $f$ of $W$ is given. Define a diffeomorphism $\tau: \boldsymbol{R} \times W$ $\rightarrow \boldsymbol{R} \times W$ by $\tau(t, x)=\left(t+1, f^{-1}(x)\right)$. Then the orbit space of $\left\{\tau^{n}: n \in Z\right\}$ is a manifold, say $W_{f}$. The flow $\psi_{t}: \boldsymbol{R} \times W \rightarrow \boldsymbol{R} \times W, \psi_{t}(u, x)=(u+t, x)$, induces a flow $\phi_{t}$ on $W_{f}$. This $\phi_{t}$ or the associated vector field to $\phi_{t}$ is called the suspension of $f$.

The suspension $\phi_{t}(x)$ of $f: W \rightarrow W$ has a cross-section $W_{0}=0 \times W \cdot \subset W_{f}$. The associated diffeomorphism with respect to $\phi_{t}$ and $W_{0}$ is trivially conjugate to the given $f$. If $\phi_{t}^{\prime}$ is the suspension of the associated 
diffeomorphism of a $C^{r}$ flow $\phi_{t}, \phi_{t}^{\prime}$ and $\phi_{t}$ are $C^{r}$ equivalent. If $f_{1}: W_{1}$ $\rightarrow W_{1}$ and $f_{2}: W_{2} \rightarrow W_{2}$ are $C^{r}$ conjugate, the suspensions of $f_{1}$ and $f_{2}$ are $C^{r}$. equivalent. These facts are formulated in [5].

Let $f$ be a $C^{r+1}$ diffeomorphism of $W$ and $X \in \mathfrak{X}^{r}\left(W_{f}\right)$ be the suspension of $f$. If $Y$ is sufficiently close to $X$ in $\mathfrak{X}^{r}\left(W_{f}\right), W_{0}$ is also a crosssection of $Y$. So, we have a map $A$ from a neighborhood of $X$ in $\mathfrak{X}^{r}\left(W_{f}\right)$ into $\mathscr{D}^{r}\left(W_{0}\right)=\mathscr{D}^{r}(W)$ by corresponding to $Y$ the associated diffeomorphism of $W_{0}=0 \times W \subset W_{f}$.

(3.1) Proposition. Let $W$ be a closed manifold, $f$ be a $C^{r+1}$ diffeomorphism of $W, r \geqq 1$, and $X$ be the suspension of $f$. Then there are neighborhoods $V$ of $X$ in $\mathfrak{X}^{r}\left(W_{f}\right)$ and $U$ of $f$ in $\mathscr{D}^{r}(W)$ such that

(i) on $V$ the map $A$ is defined and continuous,

(ii) there is a continuous map $S: U \rightarrow V$ such that $A \circ S=\mathrm{id}$, so $A(V)=U$, and

(iii) $A$ is an open map.

By (ii) it is obvious that $S$ is surjective and that for $g \in U S(g)$ is $C^{r}$ equivalent to the suspension of $g$.

Proof of (3.1). Let $V$ be a neighborhood of $X$ on which $A$ is defined. It is elementary to see that $A$ is continuous on $V$.

Let $\exp _{x}$ be the exponential map from a neighborhood of the origin in the tangent space of $W$ at $x$ onto a neighborhood of $x$ in $W$. If we define a diffeomorphism $\exp (X)$ for a vector field $X$ near the trivial field $O_{W}$ by $(\exp (X))(x)=\exp _{x}\left(X_{x}\right)$, exp is a homeomorphism from a neighborhood of $O_{W}$ in $\mathfrak{X}^{r}(W)$ onto a neighborhood $U_{0}$ of $\operatorname{id}_{W}$ in $\mathscr{D}^{r}(W)$ such that $\exp \left(O_{W}\right)=\mathrm{id} \mathrm{d}_{W}$. Put $U=\left\{f f^{\prime} \mid f^{\prime} \in U_{0}\right\}$. Let $\lambda(t):[0,1] \rightarrow[0.1]$ be a $C^{\infty}$ function such that on the neighborhoods of $0,1 \lambda(t)=0, \lambda(t)=1$ respectively. For $g \in U$ we define a $C^{r}$ diffeomorphism $h_{g}: I \times W \rightarrow I \times W$ as follows (See Figure 1)

$$
h_{g}(t, x)=\left(t, \exp \left(\lambda(t) \cdot \exp ^{-1} f^{-1} g\right)(x)\right)
$$

For $g \in U$ we define a vector field $S(g)$ on $W_{f}$ as follows. Here, we use the identifications $W_{f}=I \times W /(0, f(x)) \sim(1, x), W_{f}-W_{0}=(0,1) \times W$, and $T_{(t, x)}\left(W_{f}\right)=R \times T_{x}(W),(t, x) \in W_{f}$. 

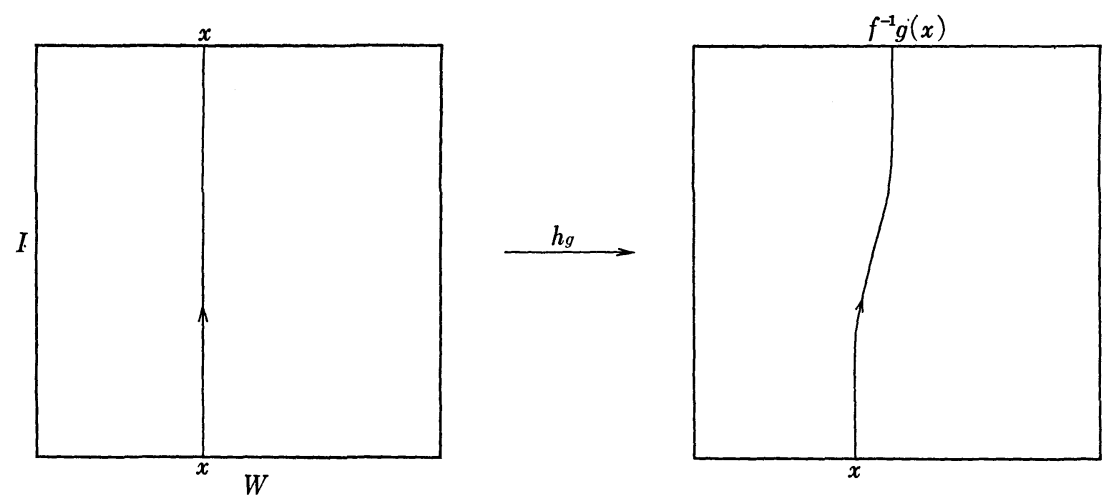

Figure 1

$$
S(g)_{(t, x)}=\left\{\begin{array}{lll}
\left(1,\left.\frac{d}{d s} h_{g}(s, x)\right|_{s=t}\right) & \text { for } & (t, x) \in W_{f}-W_{0} \\
(1,0) & \text { for } \quad(t, x) \in W_{0} .
\end{array}\right.
$$

Using the property of $\lambda$, we can see that $S(g)$ is $C^{r}$ on $W-W_{0}$ and for $(t, x)$ near $W_{0} S(g)_{(t, x)}=(1,0)$. Hence $S(g)$ is $C^{r}$. Since exp is a homeomorphism $S: U \rightarrow \mathfrak{X}^{r}\left(W_{f}\right)$ is continuous. $W_{0}$ becomes also a crosssection of $S(g)$. By using the identification $W_{f}=I \times W /(0, f(x)) \sim(1, x)$ we can show that, denoting by $\phi_{t}(x ; S(g))$ the flow associated with $S(g)$, $\phi_{1}(x ; S(g))=g(x) . \quad($ See Figure 2$)$

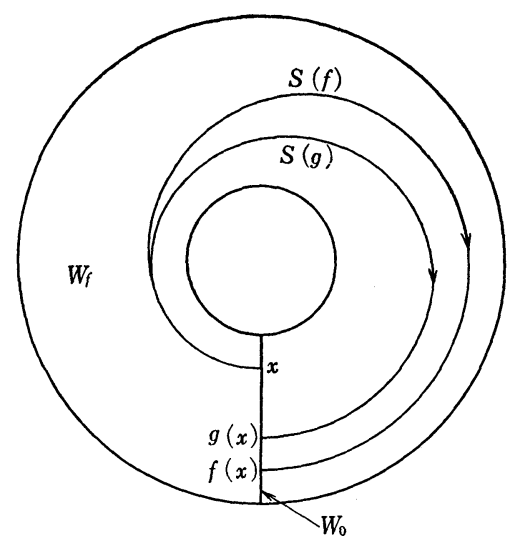

Figure 2

Next we prove (iii). We will prove that for any open subset $V^{\prime}$ of $V$ and any $g$ in $A\left(V^{\prime}\right)$ there is an open neighborhood $U^{\prime}$ of $g$ such that $A\left(V^{\prime}\right) \supset U^{\prime}$. 
$W_{f}$ is a $C^{r+1}$ manifold but it is $C^{\infty}$ outside of $W_{0}$. Let $\tilde{Y} \in V^{\prime}$ be a $C^{r}$ vector field of $W_{f}$ such that $\tilde{Y}$ induces a $C^{\infty}$ vector field, denoted also by $\tilde{Y}$, of Int $I \times W$ and that to $\tilde{g}^{-1} g \exp ^{-1}$ is defined, where $\tilde{g}=A(\tilde{Y})$.

$\tilde{Y}_{(0, x)}=\tilde{Y}_{(1, f(x))}$ by the identification id $\times T f: T_{(0, x)}(I \times W) \rightarrow T_{(1, f(x))}$ $(I \times W)$. Conversely, a $C^{r}$ vector field $Y^{\prime}$ of $I \times W$ such that $Y_{(0, x)}^{\prime}=$ ${Y^{\prime}}_{(1, f(x))}$ induces a vector field of $W_{f}$, denote it by $Y^{\prime}$ too. Let $\tilde{S}(\tilde{g})$ be the suspension of $\tilde{g}$, which is a $C^{r}$ vector field on $W_{\tilde{g}}$. There is canonically a $C^{r+1}$ diffeomorphism $H: \operatorname{Int} I \times W \rightarrow \operatorname{Int} I \times W$ such that $H$ is an equivalence between $\tilde{Y} \mid W_{f}-W_{0}$ and $\tilde{S}(\tilde{g}) \mid W_{\tilde{g}}-W_{0}$. Define a neighborhood $U^{\prime}$ of $g$ by

$$
U_{\tilde{Y}}^{\prime}=\left\{g^{\prime} \in U:\left|\left(\exp ^{-1} \tilde{g}^{-1} g^{\prime}\right)_{x}\right|<2\left|\left(\exp ^{-1} \tilde{g}^{-1} g\right)_{x}\right|\right\} .
$$

Next, we define a $C^{r}$ vector field on $W_{\tilde{g}}$. Let $t_{0}(x)$ denote the smallest positive $t$ satisfying $\phi_{t}(x) \in W_{0}$, where $\phi_{t}(x)$ is the flow associated to the vector field $H_{*}(\tilde{Y})$ induced from $\tilde{Y}$ and the diffeomorphism $H$. For $g^{\prime} \in U_{\tilde{Y}}^{\prime}$ and $(t, x) \in I \times W$ let

$$
\tilde{h}_{g^{\prime}}(t, x)=\exp \left(\lambda\left(t / t_{0}(x)\right) \exp ^{-1} \tilde{g}^{-1} g^{\prime}\right)(x) .
$$

For $x \in W$ define a path in $I \times W$ from $(0, x)$ to $\left(1, \tilde{g}^{-1} g^{\prime}(x)\right)$ by

$$
p_{x}(s)=\left(\pi \phi_{s}(x), \tilde{h}_{g^{\prime}}\left(\phi_{s}(x)\right)\right),
$$

where $\pi$ denotes the projection $I \times W \rightarrow I$. Then, a $C^{r}$ vector field $Z$ on $I \times W$ is given by

$$
Z_{(t, x)}=\left.\frac{d}{d s} p_{x}(x)\right|_{s=t_{\bar{x}}^{-1}(t)},
$$

where $t=t_{x}(s)=\pi \phi_{s}(x) . \quad Z$ induces a $C^{r}$ vector field $Z$ on $W_{\tilde{g}}-W_{0}$. By the definition,

$$
Z_{(t, x)}=\left(H_{*}(\tilde{Y})\right)_{(t, x)}+t_{x}^{\prime}(s) \cdot \lambda^{\prime}\left(\frac{t}{t_{0}(x)}\right)\left(\exp ^{-1} \tilde{g}^{-1} g^{\prime}\right) x .
$$

The associated diffeomorphism of $Z$ is $g^{\prime}$.

Define

$$
\begin{gathered}
Y^{\prime}= \begin{cases}H_{*}^{-1} Z \text { on } W_{f}-W_{0} \\
\tilde{Y} \mid W_{0} \text { on } W_{0} .\end{cases} \\
Y_{H^{-1}(t, x)}^{\prime}=Y_{H^{-1}(t, x)}+t_{x}^{\prime}(s) \cdot \lambda^{\prime}\left(t / t_{0}(x)\right)\left(H_{*}^{-1} \exp ^{-1}\left(\tilde{g}^{-1} g^{\prime}\right)\right) x, \\
t=t_{x}(s) .
\end{gathered}
$$


The associated diffeomorphism of $Y^{\prime}$ is also $g^{\prime}$.

Since we can suppose that $V^{\prime}$ is bounded, for all $\tilde{Y}$ in $V^{\prime} t^{\prime}{ }_{x}$ and $H_{*}^{-1}$ are bounded. Hence by taking $\tilde{Y}$ closely enough to $Y$, so that taking $\tilde{g}$ closely enough to $g$, we can assume that $Y^{\prime} \in V^{\prime}$ for all $g^{\prime} \in U_{\tilde{Y}}^{\prime}$. Then $U^{\prime}{ }_{\tilde{Y}}$ is the desired neighborhood $U^{\prime}$ of $g$.

This completes the proof of (3.1).

The part (i) of the following theorem in case of structural stability is well known. In case of weak stability and structural stability this theorem is mentioned in [2] without proof.

(3.2) THEOREM. Let $f$ be a diffeomorphism of class $C^{r+1}$ of a closed manifold $W, r \geqq 1$, and $X$ be the suspension of $f$. Then

(i) If $f$ is allowably (weakly or structurally) $C^{r}$ stable, $X$ is allowably (weakly or structually) $C^{r}$ stable, and

(ii) if $X$ is allowably (weakly or structurally) $C^{r}$ stable and if there is no epimorphism $\pi_{1}(W) \rightarrow Z, f$ is allowably (weakly or structurally) $C^{r}$ stable.

Proof. We shall prove in case of allowable stability. The proof of other cases are included in the following proof.

(i) Since $f$ is allowably stable, there is a neighborhood $U_{f}$ of $f$ in $\mathscr{D}^{r}(W)$ such that for a suitable $f_{0}$ in $U_{f}$ the set of all diffeomorphisms conjugate to $f_{0}$ is dense in $U_{f}$. $U_{f}$ can be assumed to be contained in $U$ of (3.1). Let $V_{X}=A^{-1}\left(U_{f}\right)$. As $A(X)=A S(f)=f, V_{X}$ is a neighborhood of $X$. Put $S\left(f_{0}\right)=X_{0} . \quad X_{0}$ is contained in $V_{X}$. We shall show that $X_{0}$ is dispersed in $V_{X}$. Let $Y$ be any vector field in $V_{X}$ and $V_{Y}^{\prime}$ be any neighborhood of $Y$ in $V_{X}$. Since $A$ is an open $\operatorname{map}, A\left(V_{Y}^{\prime}\right)$ is a neighborhood of $A(Y)$. As $f_{0}$ is dispersed, there is $f_{0}^{\prime}$ in $A(Y)$ such that $f_{0}$ and $f_{0}^{\prime}$ are topologically conjugate. We can take $X_{0}^{\prime}$ in $V_{Y}^{\prime}$ so that $A\left(X_{0}^{\prime}\right)=f_{0}^{\prime}$. Since $A\left(X_{0}\right)=A S\left(f_{0}\right)=f_{0}$ and $f_{0}$ and $f_{0}^{\prime}$ are topologically conjugate, $X_{0}$ and $X_{0}^{\prime}$ are topologically equivalent. This shows that $X_{0}$ is dispersed in $V_{X}$. Therefore, $X$ is allowably $C^{r}$ stable.

(ii) Since $X$ is allowably stable, there is a neiborhood $V_{X}$ of $X$ such that for a suitable $X_{0}$ in $V_{X}$ the set of all vector fields equivalent to $X_{0}$ is dense in $V_{X} . \quad V_{X}$ can be assumed to be contained in $V$ of (3.1). Since $A$ is an open map, $U_{f}=A\left(V_{X}\right)$ is a neighborhood of $f$. Putting $A\left(X_{0}\right)$ $=f_{0}, f_{0}$ is contained in $U_{f}$. We shall show that $f_{0}$ is dispersed in $U_{f}$. Let $g$ be any diffeomorphism in $U_{f}$ and $U_{g}^{\prime}$ be any neighborhood of $g$ 
in $U_{f}$. Since $X_{0}$ is dispersed, there is $X_{0}^{\prime}$ in $A^{-1}\left(U_{g}\right)$ such that $X_{0}^{\prime} \sim X_{0}$. $f_{0}^{\prime}=A\left(X_{0}^{\prime}\right)$ is contained in $U_{g}$. In [1] it is proved that if there is no epimorphism $\pi_{1}(W) \rightarrow Z$, the equivalence of two flows with cross-sections which are diffeomorphic to $W$ implies the equivalence of the associated diffeomorphisms. Hence, by $X_{0} \sim X_{0}^{\prime}$ we have $f_{0}=A\left(X_{0}\right)$ and $f_{0}^{\prime}=A\left(X_{0}^{\prime}\right)$ are topologically equivalent. This shows that $f_{0}$ is dispersed in $U_{f}$. Therefore, $f$ is allowably $C^{r}$ stable.

\section{§4. Nondensity of allowable $\Omega$-stability.}

In [2] it is shown that for a compact smooth manifold $M$ with dimension $\geqq 2$ and for $2 \leqq r<\infty$ the set of all weakly $C^{r}$ stable diffeomorphisms is never dense in $\mathscr{D}^{r}(M)$.

In this section we show nondensity of allowable $\Omega$-stability on 3 torus $T^{3}$ by using the following Simon's result. In [2], by using this result, nondensity of weak $\Omega$-stability is shown, and moreover, it is shown that there is not a countable set $\left\{h_{j}\right\}$ and a Baire subset $B$ in $\mathscr{D}^{r}\left(T^{3}\right)$ such that each $f$ in $B$ is equivalent in the sense of $\simeq_{\Omega}$ to some $h_{j}$, where the notation $\simeq{ }_{\Omega}$ means $\simeq(\S 2)$ derived from $\sim_{\Omega}$ in place of $\sim$.

In [4] C. P. Simon shows

(4.1). For $f \in \mathscr{D}^{r}\left(T^{3}\right)$ and a positive integer $n, 1 \leqq r$, let $N_{n}(f)=$ number of fixed points of $f^{n}$. Then, there exists an open set $U$ in $\mathscr{D}^{r}\left(T^{3}\right)$ such that if $f_{1} \in U$ and $U_{1}$ is any neighborhood of $f_{1}$ in $U$, there is $f_{2} \in U_{1}$ and an integer $n$ such that $N_{n}\left(f_{1}\right) \neq N_{n}\left(f_{2}\right)$ and all periodic points of $f_{2}$ of period $\leqq n$ are hyperbolic.

(4.2) THEOREM. There exists an open set $U$ in $\mathscr{D}^{r}\left(T^{3}\right)$ such that if $f \in U, f$ is not allowably $C^{r}$ S-stable for $1 \leqq r$.

Proof. Let $U$ be the open set given in (4.1) and $f$ be any diffeomorphisms in $U$. Suppose $f$ be allowably $\Omega$-stable, then there is a neighborhood $V$ of $f$ in $U$ such that there is a dispersed diffeomorphism $f_{0}$ in $V$. But, by (4.1), there is $g$ in $V$ and $n$ such that $N_{n}\left(f_{0}\right)=N_{n}(g)$ and all periodic points of $g$ of period $n$ are hyperbolic. Hence, for all diffeomorphisms $g_{1}$ in a neighborhood of $g N_{n}\left(g_{1}\right)=N_{n}(g) \neq N_{n}\left(f_{0}\right)$, so $f_{0} \varkappa_{\Omega} g_{1}$. Therefore, $f_{0}$ is never dispersed in $V$ in the sense of $\sim_{\Omega}$. This is a contradiction. 


\section{§5. A weakly stable system which is not structurally stable.}

The aim of this section is to prove Theorem (5.7), which is shown in [2] without proof.

We can construct a $C^{r}$ vector field $X_{0}$ on $[1, \infty) \times S^{1}$ by the method of M. M. Pexioto and C. C. Pugh [3] satisfying the following property, $1 \leqq r \leqq \infty$.

(5.1) (i ) In $(i, i+1) \times S^{1}, i=1,2, \cdots$, there are only two fixed points $p_{i}$ and $q_{i}$. ( $p_{i}$ and $q_{i}$ are said to be associated.) $p_{i}$ is a hyperbolic saddle point. The stable manifold $W^{s}\left(p_{i}\right)$ of $p_{i}$ contains the only trajectory leaving $i \times S^{1}$ but failing to arrive at $(i+1) \times S^{1}$ and the unstable manifold $W^{u}\left(p_{i}\right)$ of $p_{i}$ intersects $(i+1) \times S^{1}$ in two points. In the region bounded by $(i+1) \times S^{1}$ and the $W^{u}\left(p_{i}\right)$ a single generic source $q_{i}$ is glued in to complete the picture. (See the Figure 3.)

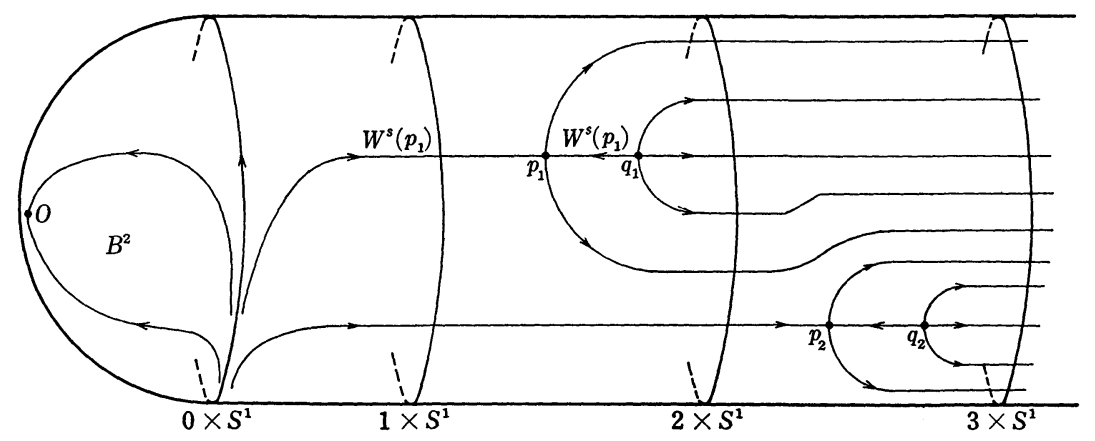

Figure 3

(ii) For any integer $k \geqq 1,\left(k \times S^{1}\right) \cap\left\{W^{s}\left(p_{i}\right) ; i=1,2, \cdots\right\}$ is a dense set in $k \times S^{1}$ and any trajectory intersects transversally with $k \times S^{1}$.

(iii) There is a $C^{1}$ neighborhood $N_{0} \subset \mathfrak{X}^{1}\left([1, \infty) \times S^{1}\right)$ of $X_{0}$ such that any $Y_{0} \in N_{0}$ has the property of (i) and (ii).

Next, we attach the boundary of a closed 2-disk $B^{2}$ to the boundary of $[0, \infty) \times S^{1}$ and regard $[0, \infty) \times S^{1} \cup B^{2}$ as the 2-plane $R^{2}$. Extend $X_{0}$ to a flow $X$ of $R^{2}$ so that $X$ has a generic sink $O$ in $B^{2}$ as an only fixed point of $X$ in $B^{2}$ and that $0 \times S^{1}$ is a hyperbolic closed orbit.

Denote $[i, i+1] \times S^{1}=\Sigma_{i}$. Since $X \mid B^{2} \cup[0,1] \times S^{1}$ and $X\left\lceil\Sigma_{i}\right.$ are Morse-Smale systems, $X$ is structurally stable in a local sense. Hence, there is a $C^{1}$ neighborhood $N$ of $X$ in $\mathfrak{X}^{r}\left(\boldsymbol{R}^{2}\right)$ such that for any $Y$ in 
$N\left\{W^{s}\left(p_{i}\right) ; i=1,2, \cdots\right\}$ is dense in $\left(k \times S^{1}\right), k=1,2, \cdots$, and that $Y \mid \Sigma_{k}$, $Y \mid B^{2}$ are equivalent to $X\left|\Sigma_{k}, X\right| B^{2}$ respectively.

Let $\nu$ be an injection from the set of saddle points $\left\{p_{i}\right\}$ of $Y \in N$ into positive integers $Z_{+}$. Call $\nu$ an admissible order of $\left\{p_{i}\right\}$ if the following condition (5.2) is satisfied.

(5.2). (i ) If $\nu\left(p_{i}\right)<\nu\left(p_{j}\right), W^{s}\left(p_{i}\right) \cap\left(W^{u}\left(p_{j}\right) \cup W^{u}\left(q_{j}\right)\right)=\phi$.

(ii) $\nu\left(p_{i}\right) \leqq i$.

(iii) $\nu^{-1}$ (i) is a finite set for any $i \in Z_{+}$, the positive integers.

Two flows $X_{0}$ and $X_{1}$ on a manifold $M$ are said to be isotopic, if there is a level preserving homeomorphism $h: I \times M \rightarrow I \times M$ such that, denoting $h(t, x)=h_{t}(x), h_{0}=\mathrm{id}$. and $h_{1}$ is an equivalence between the flows $X_{0}$ on $1 \times M$ and $X_{1}$ on $1 \times M$. $h$ is called an isotopy from $X_{0}$ to $X_{1}$.

(5.3) LEMma. Let $Y \in N,\left\{p_{i}\right\}$ and $\left\{q_{i}\right\}$ be the sets of the saddle points and sources of $Y$, and $\nu$ be an admissible order of $\left\{p_{i}\right\}$. Then, there is a flow $Z$ on $\boldsymbol{R}^{2}$ satisfying the followings. There is an isotopy from $Y$ to $Z$. By the isotopy $p_{i}$ and $q_{i}$ are brought to a saddle and a source of $Z$ contained in $\left(\nu\left(p_{i}\right)+\frac{1}{3}\right) \times S^{1}$ and $\left(\nu\left(p_{i}\right)+\frac{2}{3}\right) \times S^{1}$ respectively, where in $Y p_{i}$ and $q_{i}$ are assumed to be contained in $\Sigma_{i}$.

Proof. We shall construct a flow $Z_{i}$ by repeating isotopical modifications from $Z_{0}=Y$ inductively, where we shall make isotopies so that $Z_{k}=Z_{k-1}$ on $B^{2} \cup \Sigma_{0} \cup \cdots \cup \Sigma_{k-1}$.

Let $\nu\left(p_{i}\right)=1$, by (5.2) (ii) such $p_{i}$ exists. Bring $p_{i}$ and $q_{i}$ into $\Sigma_{1}$ by an isotopy of $Y$ so that any other fixed points of $Y$ are not changed. In fact we can do this; since $\nu\left(p_{i}\right)<\nu\left(p_{j}\right)$ for any $p_{j}(j \neq i)$, (5.2) (i) guarantees that there is no saddle point or source of $Y$ in $\Sigma_{2} \cdots \Sigma_{i}$ which obstructs to bring $p_{i}$ and $q_{i}$ into $\Sigma_{1}$ by an above isotopy. Next, we can bring all saddles $q$ with the addmissible order $1(\nu(q)=1)$ and all corresponding sources into $\Sigma_{1}$ by an isotopy so that any other fixed points are not changed and that all saddles and sources in $\Sigma_{1}$ are contained in $\left(1+\frac{1}{3}\right) \times S^{1}$ and $\left(1+\frac{2}{3}\right) \times S^{1}$ after the variation. We denote the resulting flow on $\boldsymbol{R}^{2}$ by $Z_{1}$ and denote all saddles and sources of $Z_{1}$ in $\Sigma_{1} \cup \Sigma_{2} \cup \ldots$ by $\left\{p_{i}\right\}$ and $\left\{q_{i}\right\}$ respectively, where we consider $p_{i}$ or $q_{i}$ of $Z_{1}$ to be the result of the modifications of $p_{i}$ or $q_{i}$ of $Y$ respectively.

Assume we have constructed a flow $Z_{k-1}$ on $R^{2}$ so that $Y=Z_{0}$ is 
isotopio to $Z_{k-1}$ by an isotopy which does not change $Y \mid B^{2} \cup \Sigma_{0}$ and any $p_{i}, q_{i}$ with $\nu(i) \geqq k$ and that in $Z_{k-1} p_{i} \in\left(\nu(i)+\frac{1}{3}\right) \times S^{1}, q_{i} \in\left(\nu(i)+\frac{2}{3}\right) \times S^{1}$ for $\nu(i)<k$. For all $i \geqq k$, if $p_{i} \in \Sigma_{i}$ in $Z_{k-1}, \nu\left(p_{i}\right) \geqq k$.

Similarly as we construct $Z_{1}$ from $Z_{0}$, we can change $Z_{k-1}$ to construct $Z_{k}$ by an isotopy on $\Sigma_{k} \cup \Sigma_{k+1} \cup \cdots$. In $Z_{k}$ all saddles with admissible order $k$ are contained in $\left(k+\frac{1}{3}\right) \times S^{1}$ and all corresponding sources are contained in $\left(k+\frac{2}{3}\right) \times S^{1}$.

By the above construction of $Z_{k}, \lim _{k \rightarrow \infty} Z_{k}$ converges to a flow $Z$ which is isotopic to $Y$. This $Z$ is the desired one.

Let $p$ be a saddle in $[1, \infty) \times S^{1}$. Fix an orientation of $S^{1}$. Let $W_{\ell}^{u}(p)$ denote the component of the unstable manifold $W^{u}(p)$ of $p$ which go out from $p$ with the same orientation as $S^{1}$ when we set the flow near $p$ in standard situation by an isotopy, and let $W_{r}^{u}(p)$ denote the another part of $W^{u}(p)$. (See Figure 4.)

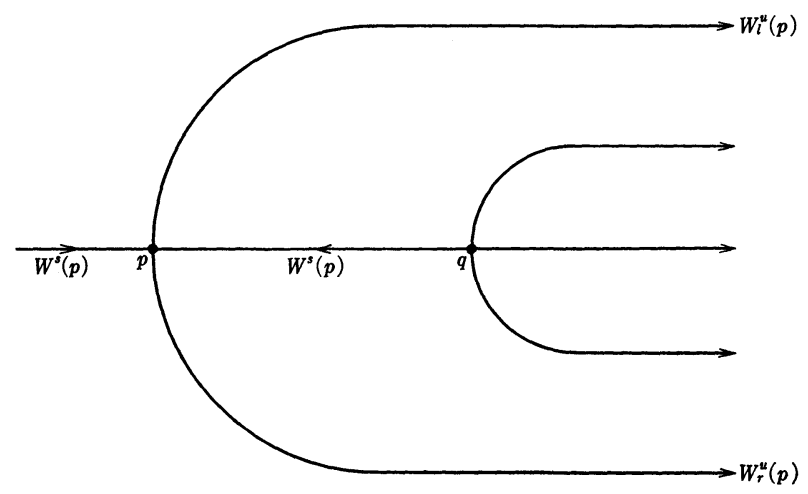

Figure 4

Let $\pi ;[0, \infty) \times S^{1} \rightarrow[0, \infty)$ be the projection onto the first factor. For dynamical systems in the open set $N$ we define an equivalence relation as follows. If $Y$ and $Y^{\prime}$ are equivalent in the following sense, $W(Y)$ and $W\left(Y^{\prime}\right)$ will be homeomorphic (lemma (5.5)). Here, $W(Y)$ denotes the subset of $R^{2}$ that consists of all stable and unstable manifolds of all saddle points of $Y$.

(5.4) Definition. Two dynamical systems $Y$ and $Y^{\prime}$ contained in $N$ are geometrically equivalent if the following conditions are satisfied.

(i) For the set $\left\{p_{i}\right\}$ and $\left\{p_{i}^{\prime}\right\}$ of all saddles of $Y$ and $Y^{\prime}$ respectively there are admissible orders, which we denote by the same symbol $\nu$. 
(ii) There is an invariant bijection $f ;\left\{p_{i}\right\} \rightarrow\left\{p_{i}^{\prime}\right\}$ with respect to $\nu$, i.e. $\nu f\left(p_{i}\right)=\nu\left(p_{i}\right)$.

(iii) For any saddle point $p$ of $Y$ let $f(p)=p^{\prime}$. By an isotopy we change $Y$ to a flow $Y_{p}$ so that, for any saddle point $p_{i}$ with $\nu\left(p_{i}\right) \leqq \nu(p)$ and $p_{i} \neq p, \pi\left(p_{i}\right)<\pi(p)$ and $\pi(p) \times S^{1} \cap W^{u}\left(p_{i}\right)$ is a set containing only two points; by lemma (5.3) this is possible. Similarly change $Y^{\prime}$ to $Y^{\prime}{ }_{p^{\prime}}$. By the above condition (ii) the numbers of the elements of the sets $\left\{p_{i} \mid \nu\left(p_{i}\right) \leqq \nu(p)\right\}$ and $\left\{p_{i}^{\prime} \mid \nu\left(p_{i}^{\prime}\right) \leqq \nu\left(p^{\prime}\right)\right\}$ are equal. The condition (iii) is the following. With respect to $Y_{p}$ and $Y_{p^{\prime}}^{\prime}$ there is an orientation preserving homeomorphism $h_{p}: \pi(p) \times S^{1} \rightarrow \pi\left(p^{\prime}\right) \times S^{1}$ such that $h_{p}(p)=p^{\prime}$ and that for any $p_{i}$ with $\nu\left(p_{i}\right) \leqq \nu(p)$ and $p_{i} \neq p$ the following are satisfied.

$$
h_{p}\left(W_{\ell}^{u}\left(p_{i}\right) \cap \pi(p) \times S^{1}\right)=W_{\ell}^{u}\left(f\left(p_{i}\right)\right) \cap \pi\left(p^{\prime}\right) \times S^{1},
$$

and

$$
h_{p}\left(W_{r}^{u}\left(p_{i}\right) \cap \pi(p) \times S^{1}\right)=W_{r}^{u}\left(f\left(p_{i}\right)\right) \cap \pi\left(p^{\prime}\right) \times S^{1} .
$$

Condition (iii) is independent of the choice of $Y_{p}$ and $Y_{p^{\prime}}^{\prime}$ or the choice of isotopies. If saddle points $\left\{p_{i} \mid \nu\left(p_{i}\right) \leqq \nu(p)\right\}$ and $\left\{p_{i}^{\prime} \mid \nu\left(p_{i}^{\prime}\right) \leqq \nu\left(p^{\prime}\right)\right\}$ of two flows in $N$ can be situated by isotopies as these in $Y_{p}$ and $Y_{p^{\prime}}^{\prime}$ of (iii) $p$ and $p^{\prime}$ are said to have the same situation with $\left\{p_{i} \mid \nu\left(p_{i}\right) \leqq \nu(p)\right.$, $\left.p_{i} \neq p\right\}$ and $\left\{p_{i}^{\prime} \mid \nu\left(p_{i}^{\prime}\right) \leqq \nu\left(p^{\prime}\right), p_{i}^{\prime} \neq p^{\prime}\right\}$.

(5.5) LEMMA. For any two flows $Y$ and $Y^{\prime}$ in $N$ and any neighborhood $U$ of $Y^{\prime}$ in $\mathscr{D}^{r}\left(\boldsymbol{R}^{2}\right), 1 \leqq r \leqq \infty$, there is a $C^{\infty}$ flow $Z$ in $U$ such that $Y$ and $Z$ are geometrically equivalent.

Proof. Starting from $Y^{\prime}=Z$, we will construct $Z_{k}$ inductively by perturbations step by step. In the construction a positive integer $j(k)$ is defined for all integer $k$ so that $k<j(k)<j(k+1)$. $\quad Z_{k}$ will be contained from $Z_{k-1}$ by a perturbation only in $\Sigma_{k} \cup \Sigma_{j(k)}$ and only outside of neighborhoods of fixed points of $Z_{k-1}$ in $\Sigma_{k} \cup \Sigma_{j(k)} . \quad Z$ will be defined as $\lim _{k \rightarrow \infty} Z_{k}$. Since $Z=Z_{k}$ for all $k$ in a neighborhoods of fixed points, we may assume that the set of the saddle points of $Z_{k}$ is the same as that of $Z$. In this sense an admissible order $\nu$ and a bijection $f$ will be defined on an increasing sequence of subsets of the saddle points of $Y$ or $Z_{k}$ at the same time when we define $Z_{k}$ inductively.

Let $p_{i}$ and $p_{i}^{\prime}$ denote the saddle points of $Y$ and $Z_{k}$ contained in $\Sigma_{i}$.

Step 1. Let $Y^{\prime}=Z_{1}$. We define $\nu\left(p_{1}\right)=1, \nu\left(p_{1}^{\prime}\right)=1$, and $f\left(p_{1}\right)=p_{1}^{\prime}$. 
Step 2. Define $\nu\left(p_{2}\right)=2$. (a) If $p_{2}$ in $Y$ and $p_{2}^{\prime}$ in $Z_{1}$ have the same situation with $p_{1}$ and $p_{1}^{\prime}$ we define $Z_{2}=Z_{1}, \nu\left(p_{2}^{\prime}\right)=2$, and $f\left(p_{2}\right)=p_{2}^{\prime}$. (b) The case that $p_{2}$ in $Y$ and $p_{2}^{\prime}$ in $Z_{1}$ have not the same situation with $p_{1}$ and $p_{1}^{\prime}$. We can take an integer $i(2)>2$ and a perturbation $Z_{2}^{\prime}$ of $Z_{1}$ only on $\Sigma_{2}$ keeping fixed on neighborhoods of fixed points in $\Sigma_{2}$ so that in $Y$ and $Z_{2}^{\prime} p_{i(2)}$ and $p_{2}^{\prime}$ have the same situation with $p_{1}$ and $p_{1}^{\prime}$. This perturbation of $Z_{1}$ is necessary only in case that $W^{u}\left(p_{1}^{\prime}\right) \cap W^{s}\left(p_{2}^{\prime}\right) \neq \phi$ but $W^{u}\left(p_{1}\right)$ does not intersect with any stable manifold of other saddle or the case that $W^{u}\left(p_{1}^{\prime}\right) \cap W^{s}\left(p_{2}^{\prime}\right)=\phi$ but $W_{\ell}^{u}\left(p_{1}\right)$ and $W_{r}^{u}\left(p_{1}\right)$ are contained in stable manifolds of saddle points. Here we define $\nu\left(p_{i(2)}\right)=2, \nu\left(p_{2}^{\prime}\right)=2$, and $f\left(p_{i(2)}\right)=p_{2}^{\prime}$. Next, as above, we can take an integer $j(2)>2$ and a perturbation $Z_{2} \in U$ of $Z_{2}^{\prime}$ only on $\Sigma_{j(2)}$ so that $p_{2}$ and $p_{j(2)}^{\prime}$ have the same situation with $\left\{p_{1}, p_{i(2)}\right\}$ and $\left\{p_{1}^{\prime}, p_{2}^{\prime}\right\}$. Here we define $\nu\left(p_{j(2)}^{\prime}\right)=2$ and $f\left(p_{2}\right)=p_{j(2)}^{\prime}$.

We assume that we have defined inductively $Z_{k-1}$ and $\nu, f$ on subsets of saddle points of $Y$ and $Z_{k-1}$, i.e. we assume we have finished step $(k-1)$.

Step $k$. (a) In the case that $p_{k}=p_{i(m)}$ and $p_{k}^{\prime}=p_{j(n)}^{\prime}$ for suitable $m, n<k$, we put $Z_{k}=Z_{k-1}$. In this case there is no saddle point which has admissible order $k$.

(b) The case that $p_{k} \neq p_{i(m)}$ and $p_{k}^{\prime} \neq p_{j(n)}^{\prime}$ for any $m, n<k$ and that $p_{k}$ and $p_{k}^{\prime}$ have the same situation with all saddle points of $Y$ and $Z_{k-1}$ to which admissible orders $<k$ are defined in all the previous steps. In this case we define $Z_{k}=Z_{k-1}, \nu\left(p_{k}\right)=k, \nu\left(p_{k}^{\prime}\right)=k$, and $f\left(p_{k}\right)=p_{k}^{\prime}$.

(c) The other case. If $p_{k}^{\prime}=p_{j(n)}^{\prime}$ for a $n<k, \nu$ and $f$ are defined for $p_{k}^{\prime}$ in a previous step. In this case put $Z_{k}^{\prime}=Z_{k-1}$. In the another case we can take an integer $i(k)>k$ and a perturbation $Z_{k}^{\prime} \in U$ of $Z_{k-1}$ only on $\Sigma_{k}$ as above so that $p_{i(k)}$ and $p_{k}^{\prime}$ have the same situation with all saddle points to which admissible orders $<k$ are defined in all the previous steps. Here, we define $\nu\left(p_{i(k)}\right)=k, \nu\left(p_{k}^{\prime}\right)=k$, and $f\left(p_{i(k)}\right)=p_{k}^{\prime}$. The above perturbation should be taken so small that the condition (5.2) (i) is not broken for all saddle points in $Z_{k-1}^{\prime}$ to which admissible orders defined in previous steps.

If $p_{k}=p_{i(m)}$ for a $m<k, \nu$ and $f$ are defined for $p_{k}$ in a previous step. In this case put $Z_{k}=Z_{k}^{\prime}$. In the another case we can take an integer $j(k)>k$ and a perturbation $Z_{k} \in U$ of $Z_{k}^{\prime}$ only on $\Sigma_{j(k)}$ as above so that $p_{k}$ and $p_{j(k)}^{\prime}$ have the same situation with all saddle points to 
which admissible order $\leqq k$ are defined before. Here we define $\nu\left(p_{k}\right)=k$, $\nu\left(p_{j(k)}^{\prime}\right)=k$, and $f\left(p_{k}\right)=p_{j(k)}^{\prime}$.

By the above construction there exists $Z=\lim _{k \rightarrow \infty} Z_{k}$ and to all saddle points of $Y$ and $Z$ admissible order $\nu$ is defined. And by the construction $Y$ and $Z$ are geometrically equivalent. This proves (5.5).

(5.6) LEMMA. If $Y$ and $Y^{\prime}$ in $N$ are geometrically equivalent, there exists a homeomorphism $h$ from $W(Y)$ onto $W\left(Y^{\prime}\right)$ preserving the orientations of trajectories with the following properties. (i) $h\left(\left(i \times S^{1}\right) \cap\right.$ $W(Y))=\left(i \times S^{1}\right) \cap W\left(Y^{\prime}\right), i=0,1,2, \cdots$, and (ii) if $f\left(p_{i}\right)=p_{j}^{\prime}$, then $h\left(p_{i}\right)=p_{j}^{\prime}, h\left(q_{i}\right)=q_{j}^{\prime}, h\left(W^{s}\left(p_{i}\right)\right)=W^{s}\left(p_{j}^{\prime}\right), h\left(W_{\ell}^{u}\left(p_{i}\right)\right)=W_{b}^{u}\left(p_{j}^{\prime}\right), h\left(W_{r}^{u}\left(p^{i}\right)\right)=$ $h\left(W_{r}^{u}\left(p_{j}^{\prime}\right)\right)$, and $h\left(W^{u}\left(q_{i}\right)\right)=W^{u}\left(q_{j}^{\prime}\right)$. Here, $f:\left\{p_{i}\right\} \rightarrow\left\{p_{j}^{\prime}\right\}$ is the bijection which produces the geometrial equivalence.

Proof. It is sufficient to prove (5.6) for $Z$ and $Z^{\prime}$ that are obtained by isotopies from $Y$ and $Y^{\prime}$ using lemma (5.3). Let $\left\{p_{k_{(1)}}, \cdots, p_{k_{(j)}}\right\}$ be the set of all saddle points of $Z$ contained in $\Sigma_{k}$. Taking a small perturbation of $Z$ by an isotopy $Z$ can be assumed to have the following properties.

(a) The trajectories of $Z$ are transversal to $k \times S^{1}$ for $k=1,2, \cdots$, transversal to $t \times S^{1}$ for $t \in(0,1)$, and transversal to $S_{r}^{1}$, where $S_{r}^{1}$ is the any circle with origin $O$ and radius $r$ contained in $\operatorname{Int} B^{2}$.

(b) Every trajectory that is not contained in $\left(W^{u}\left(q_{k_{(1)}}\right) \cup \cdots \cup\right.$ $\left.W^{u}\left(q_{k(j)}\right)\right) \cup\left(W^{u}\left(p_{k(1)}\right) \ldots W^{u}\left(p_{k(j)}\right)\right)$ is transversal to $t \times S^{1}$ for $k<t<$ $k+1$.

(c) For any saddle point $p \in \Sigma_{k}$ and any $t \in[k, k+1]\left(W^{u}(p)-\{p\}\right)$ is transversal to $t \times S^{1}$.

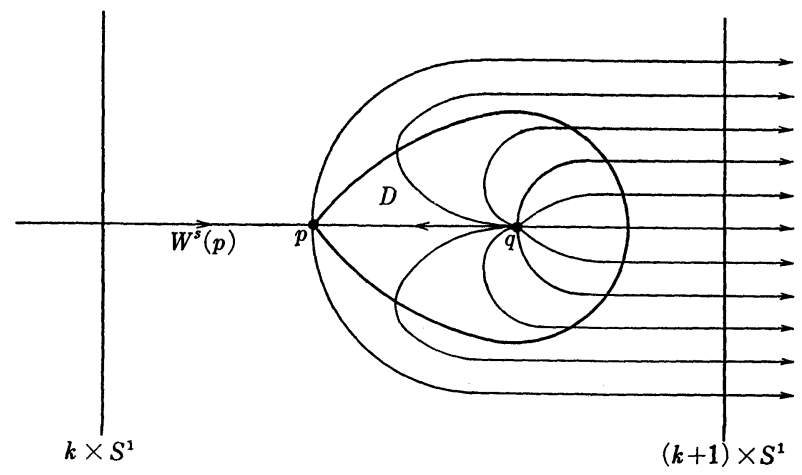

Figure 5 
(d) For any source $q \in \Sigma_{k}$ there is a closed disk $D$ such that $q \in \operatorname{Int} D \subset W^{u}(q) \cap \Sigma_{k}$, denoting by $p$ the associated saddle point with $q D \cap W^{u}(p)=p, \partial D-\{p\}$ is smooth, and every trajectory in $W^{u}(q)$ is transversal to $\partial D$. (See Figure 5.)

Similarly we bring $Z^{\prime}$ to the above situation.

Let $m \geqq 1$ be an integer. We define a map $h_{m}: W(Z) \cap\left(m \times S^{1}\right) \rightarrow$ $W\left(Z^{\prime}\right) \cap\left(m \times S^{1}\right)$ as follows; for $a \in W(Z) \cap\left(m \times S^{1}\right)$ define

$$
h_{m}(a)= \begin{cases}W^{s}(f(p)) \cap m \times S^{1} & \text { if } a \in W^{s}(p) \text { for a saddle } p \\ W_{\ell}^{u}(f(p)) \cap m \times S^{1} & \text { if } a \in W_{\ell}^{u}(p) \\ W_{r}^{u}(f(p)) \cap m \times S^{1} & \text { if } a \in W_{r}^{u}(p)\end{cases}
$$

where $f:\left\{p_{i}\right\} \rightarrow\left\{p_{i}^{\prime}\right\}$ is the bijection from the set of saddle points of $Z$ onto that of $Z^{\prime}$ as above. $h_{m}$ is well defined, for $p \in \Sigma_{i}$ implies $f(p) \in \Sigma_{i}$ by the construction of $Z$ and $Z^{\prime}$.

Next we show that $h_{m}$ is a homeomorphism. Let $a_{1}, a_{2}, a_{3} \in W(Z) \cap$ $\left(m \times S^{1}\right)$ and suppose that $a_{1}, a_{2}, a_{3}$ are ordered in this way with the orientation of $S^{1}$. Let $a_{k} \in W\left(p_{i_{k}}\right)$, where $k=1,2,3$ and $W\left(p_{i_{k}}\right)=W^{s}\left(p_{i_{k}}\right)$ $\cup W^{u}\left(p_{i_{k}}\right)$. Let $p$ be a saddle of $Z$ such that $\pi(p)$ is larger than $\pi\left(p_{i_{1}}\right)$, $\pi\left(p_{i_{2}}\right)$, and $\pi\left(p_{i_{3}}\right)$. Then there is an orientation preserving continuous surjection $g: \pi(p) \times S^{1} \rightarrow m \times S^{1}$ such that (a) for $x \in m \times S^{1} g^{-1}(x)$ is one point if and only if $x \notin W^{s}\left(p_{i_{1}}\right) \cup W^{s}\left(p_{i_{2}}\right) \cup W^{s}\left(p_{i_{3}}\right)$, (b) if $x=W^{s}\left(p_{i_{k}}\right) \cap$ $m \times S^{1} g^{-1}(x)=\mathrm{Cl} W^{u}\left(q_{i_{k}}\right) \cap \pi(p) \times S^{1}$, and that (c) if $x=W_{\ell}^{u}\left(p_{i_{k}}\right) \cap m \times$ $S^{1} g^{-1}(x)=W_{\ell}^{u}\left(p_{i_{k}}\right) \cap \pi(p) \times S^{1}$, and if $x=W_{r}^{u}\left(p_{i_{k}}\right) \cap m \times S^{1} g^{-1}(x)=W_{r}^{u}\left(p_{i_{k}}\right)$ $\cap \pi(p) \times S^{1}$. (See Figure 6.)

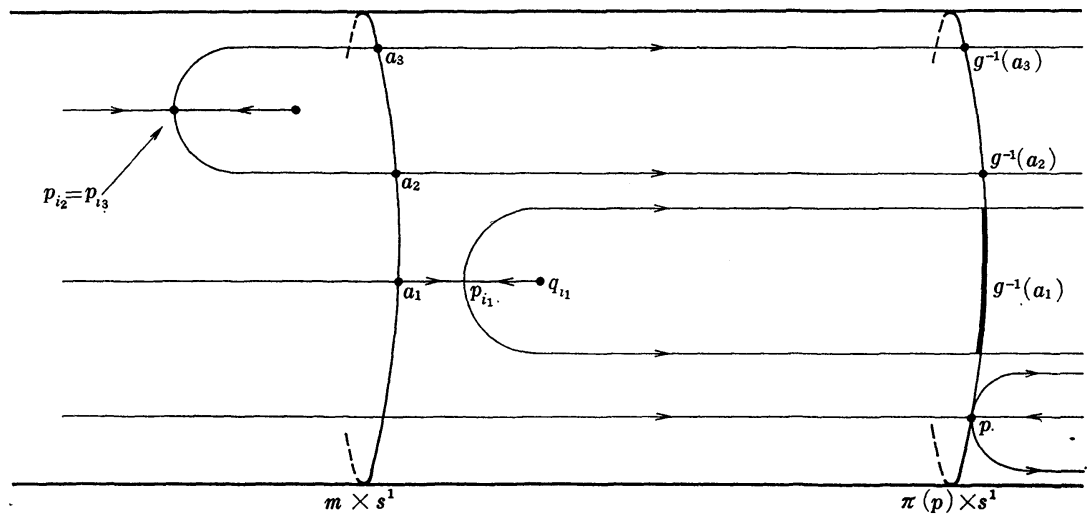

Figure 6 
Put $h_{m}\left(a_{k}\right)=a_{k}^{\prime}, k=1,2,3$. For $Z^{\prime}$ there is a map $g^{\prime}: \pi(f(p)) \times S^{1} \rightarrow$ $m \times S^{1}$ as above. Since $p$ and $f(p)$ have the same situation with $\left\{p_{i_{1}}, p_{i_{2}}\right.$, $\left.p_{i_{3}}\right\}$ and $\left\{f\left(p_{i_{1}}\right), f\left(p_{i_{2}}\right), f\left(p_{i_{3}}\right)\right\}, \quad p$ and $f(p)$ are situated in $p \times S^{1}$ and $f(p) \times S^{1}$ in the same order with $\left\{g^{-1}\left(a_{1}\right), g^{-1}\left(a_{2}\right), g^{-1}\left(a_{3}\right)\right\}$ and $\left\{g^{\prime-1}\left(a_{1}^{\prime}\right)\right.$, $\left.g^{\prime-1}\left(a_{2}^{\prime}\right), g^{\prime-1}\left(a_{3}^{\prime}\right)\right\}$, respectively. Hence, $a_{1}, a_{2}, a_{3}$ and $a_{1}^{\prime}, a_{2}^{\prime}, a_{3}^{\prime}$ are ordered in the same way with respect to the orientation of $S^{1}$. Since $a_{1}, a_{2}$, and $a_{3}$ are arbitrary points of $W(Z) \cap\left(m \times S^{1}\right), h_{m}$ preserves the orders of the points in $W(Z) \cap\left(m \times S^{1}\right)$ and $W\left(Z^{\prime}\right) \cap\left(m \times S^{1}\right)$ with respect to the orientation of $S^{1}$. As $W(Z) \cap\left(m \times S^{1}\right)$ and $W\left(Z^{\prime}\right) \cap\left(m \times S^{1}\right)$ are dense subsets of $m \times S^{1}$, this implies $h_{m}$ is continuous. Since $h_{m}$ is bijective, $h_{m}$ is a homeomorphism.

Next, we define a homeomorphism $h: W(Z) \rightarrow W\left(Z^{\prime}\right)$.

Let $W(x)$ and $W^{\prime}(x)$ be the trajectories of $Z$ and $Z^{\prime}$ passing on $x$. If $x \in W(Z) \cap \Sigma_{k}-\mathrm{Cl}\left(W^{u}\left(q_{k(1)}\right) \cup \cdots \cup W^{u}\left(q_{k(j)}\right)\right), k=1,2, \cdots$, we define $h(x)=W^{\prime}\left(h_{k}\left(W(x) \cap k \times S^{1}\right)\right) \cap \pi(x) \times S^{1}$. If $x \in W_{\ell}^{u}(p)$ or $W_{r}^{u}(p)$ for a saddle $p$ of $Z$ in $\Sigma_{k}$, we define $h(x)=W_{\ell}^{u}(f(p)) \cap \pi(x) \times S^{1}$ or $W_{r}^{u}(f(p)) \cap$ $\pi(x) \times S^{1}$ respectively. By the properties (b), (c) of $Z$ and $Z^{\prime}, h$ is well defined for these $x$ and $h(p)=f(p)$ for a saddle $p$. Let $q \in \Sigma_{k}$ be a source. On $W(Z) \cap W^{u}(q), h$ is defined as follows. Let $p$ be the saddle in $\Sigma_{k}$ associated with $q$, and $q^{\prime}$ be the source of $Z^{\prime}$ associated with $h(p)$. Let $D$ and $D^{\prime}$ be the disks given in (d) above containing $q$ and $q^{\prime}$ respectively. For $a \in \partial D \cap W(Z)$ we define $h(a)=W^{\prime}\left(h\left(W(a) \cap(k+1) \times S^{1}\right)\right)$ $\cap D^{\prime}$. We can extend $h$ over $W(Z) \cap D$ so that $h$ maps $W(Z) \cap D$ homeomorphically onto $W\left(Z^{\prime}\right) \cap D^{\prime}$ and $h(q)=q^{\prime}$. We can extend $h$ over $W(Z) \cap\left(W^{u}(q) \cap \Sigma_{k}-D\right)$ so that $h$ maps $W(Z) \cap\left(W^{u}(q) \cap \Sigma_{k}-D\right)$ homeomorphically onto $W\left(Z^{\prime}\right) \cap\left(W^{u}\left(q^{\prime}\right) \cap \Sigma_{k}-D^{\prime}\right)$. Therefore, we have defined a homeomorphism $h: W(Z) \cap[1, \infty) \times S^{1} \rightarrow W\left(Z^{\prime}\right) \cap[1, \infty) \times S^{1}$.

Finally, by extending $h$ over $\Sigma_{0}$ we have a homeomorphism $h: W(Z)$ $\rightarrow W\left(Z^{\prime}\right)$. This proves (5.6).

(5.7) THEOREM. For $1 \leqq r \leqq \infty$ there exists an open set $N$ of $\mathfrak{X}^{r}\left(\boldsymbol{R}^{2}\right)$ such that

(i) any vector field contained in $N$ is not structurally $C^{r}$ stable,

(ii) any vector field in $N$ is weakly $C^{r}$ stable and $C^{r} \Omega$-stable, and

(iii) $N$ contains uncountably many equivalence classes of vector fields but $X \simeq Y$ for any $X, Y \in N$.

Proof. Let $X_{0}$ and $N$ be the vector fields on $R^{2}$ and the neighborhood 
of $X_{0}$ given in the first place of this section. The $\Omega$-stability of $X \in N$ is clear from the fact that $X \mid B^{2}$ and $X \mid \Sigma_{k}, k=0,1,2, \cdots$, are MorseSmale. For $X \in N$ we can find $Y$ arbitrarily near to $X$ so that $X$ has two saddle points $p$ and $p^{\prime}$ with $W^{s}(p) \cap W^{u}\left(p^{\prime}\right) \neq p$. But such $Y$ is not structurally stable. So, $X$ is not structurally stable.

Next we show that any vector field in $N$ is weakly $C^{r}$ stable. Let $Y, Y^{\prime} \in N$. In any neighborhood $U$ of $Y^{\prime}$ there is $Y^{\prime \prime}$ such that $Y$ and $Y^{\prime \prime}$ are geometrically equivalent by lemma (5.5). Then, by lemma (5.6), we have a homeomorphism $h: W(Y) \rightarrow W\left(Y^{\prime \prime}\right)$ satisfying the properties (i), (ii) in (5.6). Taking small perturbations by isotopies, $Y$ and $Y^{\prime \prime}$ can be assumed to have the properties (a), (b), and (c) in the proof of (5.6). For any $t \in[1, \infty)$, let $k \leqq t<k+1$ for an integer $k$. Let $U_{k}$ and $U_{k}^{\prime \prime}$ denote the set of all points in the stable manifolds of all sources in $\Sigma_{k}$ of $Y$ and $Y^{\prime \prime}$. By the proof of (5.6) we may assume that $h(W(Y) \cap t \times$ $\left.S^{1}-U_{k}\right) \subset W\left(Y^{\prime \prime}\right) \cap t \times S^{1}-U_{k}^{\prime \prime}$. Denote $h_{t}=h \mid\left(W(Y) \cap t \times S^{1}-U_{k}\right)$. Since $W(Y)$ and $W\left(Y^{\prime \prime}\right)$ are dense in $t \times S^{1}, h_{t}$ is extended uniquely to a homeomorphism $\tilde{h}_{t}:\left(W(Y) \cap t \times S^{1}-U_{k}\right) \rightarrow\left(W\left(Y^{\prime \prime}\right) \cap t \times S^{1}-U_{k}^{\prime \prime}\right) . \quad \tilde{h}_{t}$ defines a homeomorphism $\tilde{h}:\left(\Sigma_{k}-U_{k}\right) \rightarrow\left(\Sigma_{k}-U_{k}^{\prime \prime}\right)$. Let $q$ be a source of $Y$ in $\Sigma_{k}$ and $D, D^{\prime \prime}$ be the disks as in the proof of (5.6) containing $q, h(q)$ respectively. Similarly we can define homeomorphisms $\tilde{h}: D \rightarrow D^{\prime \prime}$ and $\tilde{h}:\left(W^{u}(q) \cap \Sigma_{k}-D\right) \rightarrow\left(W^{u}(h(q)) \cap \Sigma_{k}-D^{\prime}\right)$. From these $\tilde{h}$ we have a homeomorphism $\tilde{h}: \Sigma_{k} \rightarrow \Sigma_{k}$. Hence, we have a homeomorphism $\tilde{h}:(0, \infty) \times S^{1} \rightarrow(0, \infty) \times S^{1}$ which is an extension of $h$. Extend $\tilde{h}$ to $\tilde{h}: \boldsymbol{R}^{2} \rightarrow \boldsymbol{R}^{2}$ so that $\tilde{h} \mid B^{2}$ maps the trajectory of $Y \mid B^{2}$ onto a trajectory of $Y^{\prime \prime} \mid B^{2}$. Since the one parameter subgroups $\varphi_{t}, \varphi_{t}^{\prime \prime}$ of $Y, Y^{\prime \prime}$ are families of homeomorphisms on $R^{2}$ which vary continuously with $t$, and since $\tilde{h}$ is orbit preserving on a dense subset of $R^{2}-B^{2}, \tilde{h}$ is orbit preserving on $R^{2}$. Therefore, $Y$ and $Y^{\prime \prime}$ is equivalent. This proves that any $Y$ in $N$ is weakly $C^{r}$ stable.

Next, we show that in the family of equivalence classes of flows in $N$ there is a subfamily which has the cardinal number of reals $\boldsymbol{R}$. For a dyadic expansion of $\alpha \in(0,1), \alpha=0 . \alpha_{1} \alpha_{2} \alpha_{3} \cdots, \alpha_{i}=0$ or 1 , we can fined a flow $X_{\alpha}$ in $N$ so that $X_{\alpha}$ satisfies the following properties. (i) There are saddle points $p_{k(0)}, p_{k(1)}, p_{k(2)} \cdots$ of $X_{\alpha}$ such that if $\alpha_{i}=0$ (or 1 ), $W_{\ell}^{u}\left(p_{k(i-1)}\right) \cap W^{s}\left(p_{k(i)}\right) \neq \phi$ (or $W_{r}^{u}\left(p_{k(i-1)}\right) \cap W^{s}\left(p_{k(i)}\right) \neq \phi$ respectively), and that $W_{r}^{u}\left(p_{k(i-1)}\right)$ (or $W_{\ell}^{u}\left(p_{k_{(i-1)}}\right)$ respectively) intersects the stable manifolds of no other saddle points. (ii) For any other saddle point $p$ than 
$p_{k(0)}, p_{k(1)}, p_{k(2)}, \cdots$ of (i) $W^{u}(p)$ intersects no other saddle of $X_{\alpha}$. If $\alpha=0 . \alpha_{1} \alpha_{2} \alpha_{3} \cdots$ and $\beta=0 . \beta_{1} \beta_{2} \beta_{3} \cdots$ are two different dyadic expansions, we can show that $X_{\alpha}$ and $X_{\beta}$ are not equivalent. This shows the part (iii) of (5.7).

This completes the proof of theorem (5.7).

Remark. In the proof of theorem (5.7) the homeomorphism $\tilde{h}$, which is the equivalence between $Y$ and $Y^{\prime \prime}$, is not taken near the identity.

The following problem is open. Are there diffeomorphisms or flows on a compact manifold which are weakly stable but not structurally stable? On the other hand such a question rises whether weak stability implies $\Omega$-stability.

\section{REFERENCES}

[1] G. Ikegami, On classification of dynamical systems with cross-sections, Osaka J. Math. Vol. 6 (1969), 419-433.

[2] G. Ikegami, On structural stability and weak stability of dynamical systems, Proceedings of International Conference on Manifolds and Related Topics in Topology, Tokyo Univ. Press, 1974 (to appear).

[ 3 ] M. M. Peixoto and C. C. Pugh, Structurally stable systems on open manifolds are never dense, Ann. of Math.Vol. 87 (1968), 423-430.

[4] C. P. Simon, On a classification of a Baire set of diffeomorphisms, Bull. AMS Vol. 77 (1971), 783-787.

[5] S. Smale, Stable manifolds for differential equations and diffeomorphisms, Ann. Scuola Norm. Sup. Pisa (3) 17 (1963), 97-116.

Nagoya University 